\title{
Bayesian statistics integrates prior information in parametric optical modeling
}

Richard Silver, Bryan Barnes, Nien Fan Zhang, and Hui Zhou

A new approach uses embedded data from reference metrology to reduce parametric correlation and improve measurement performance.

Semiconductor manufacturing requires measurements to control the size of nanoscale devices (referred to as the critical dimension: CD) within patterned layers and for monitoring the placement of new levels upon existing layers (known as overlay metrology). As device sizes continue to decrease, new approaches are required to refine these measurements. A significant body of recent research has investigated new optical technologies for CD and overlay metrology on scales of 32nm and smaller. Much of this work has focused on scatterometry and, more recently, scatterfield microscopy, a technique combining well-defined angle-resolved illumination with image-forming optics. ${ }^{1,2}$ These optical methods are of particular interest because of their nondestructive, high-throughput characteristics and their potential for excellent sensitivity and accuracy. However, their measurement uncertainties are fundamentally limited by the underlying cross-correlations between the different fit parameters, e.g., line widths and heights. ${ }^{3}$

To reduce parametric correlation and improve measurement performance and uncertainties, we have developed a Bayesian statistical approach that integrates a priori information gleaned from other measurements. This allows us to embed information obtained from reference metrology and complimentary ellipsometry of the optical constants, or to constrain the floating parametric range based on physical limits or known manufacturing variability. We have implemented this approach using scatterfield microscopy, but it applies equally to scatterometry or methods such as scanning-electron microscopy.

The scatterfield-microscopy instrument is based on a Köhler illuminated bright-field microscope, such that each point at the conjugate back focal plane maps nominally to a plane wave of illumination at the sample. ${ }^{4}$ Access to a large conjugate back

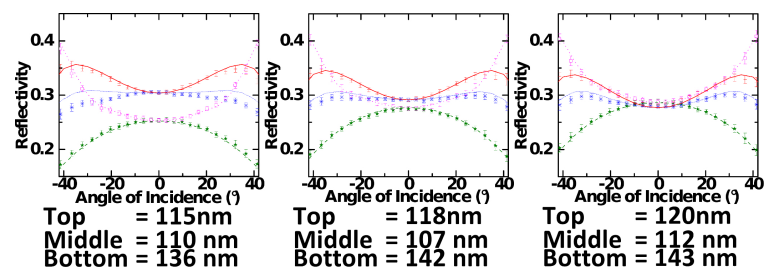

Figure 1. Experimental data and library data fits for three die from the overlay-metrology advisory group OMAG-3 wafer. Top and middle critical dimensions (CDs) show good agreement with reference values.

focal plane enables engineered illumination. This, in turn, has fueled advances in optical-system performance, characterization, and data analysis. As a result, the microscope-illumination and collection-path errors can be mapped to a functional dependence. They can then be used to normalize the experimental data for accurate comparison with electromagnetic simulations. ${ }^{5}$ We acquired both microscope images and backgrounds as a function of angle. We calculated the mean intensity of the angle-resolved images, which we corrected using the background scan that was previously normalized by the known silicon reflectance. This is similar to conventional scatterometry, except that measurements were made with high-magnification image-forming optics.

We compared the normalized experimental signatures with electromagnetic scattering simulations using parametric analysis. We assembled a library of curves by simulating a multidimensional parameter space. We completed the comprehensive simulations using a rigorous coupled-waveguide analysis (RCWA) model. ${ }^{6}$ Historically, researchers have used least-squares fitting routines to select a set of parameters with the closest experiment-to-theory agreement. ${ }^{3}$ Parametric correlation, measurement noise, and model inaccuracy all lead to measurement uncertainty in the fitting process. Even when a measurement demonstrates good sensitivity, cross-correlation

Continued on next page 
among the parameters can lead to very large uncertainties and is the fundamental limitation to the goodness of fit.

Figure 1 shows example data and fit results. They demonstrate good agreement between the simulated library of curves and the experimental data. The goodness-of-fit values and residuals are acceptable. However, the fitting process produces more uncertainty than desired, 1 to $2 \mathrm{~nm}(1 \sigma)$. In this case, the combination of parametric correlation between fit parameters and measurement noise introduces uncertainty in the final results.

To examine these correlation effects in more detail, we observed a more complicated 3D 22nm contact-hole simulation example with several layers and optical constants. We used a simulation-based analysis comparing spectroscopic and angleresolved scatterometry. ${ }^{7}$ To better understand the effects of parametric correlation, we looked at the sensitivity and covariance matrices from the $\chi^{2}$ analysis. The upper part of Figure 2 shows reflectivity data calculated for spectroscopic scans, while the middle graphs show angle-resolved scatterometry results. The uncertainties and covariance matrix are shown at bottom. The angle scans have large regions of excellent sensitivity, yet wavelength scans have smaller uncertainties. The large cross-terms in the covariance matrix lead to larger angle-scan uncertainty values for CD, sidewall, and over etch.

To decouple the parametric correlation and reduce measurement uncertainties, we developed a strategy that embeds multiple measurement uncertainties directly in the parametric fitting and uncertainty analysis. In general, a complete set of measurements consists of $N$ data points obtained under a varying set of conditions for both simulations and experiments. The $K$ model parameters are expressed as a vector $\vec{a}=\left\{a_{1}, \ldots, a_{K}\right\}$ and represent the model input parameters, for example, CD and sidewall. $N$ measured values of $Y$ are denoted as $\left\{y_{1}, \ldots, y_{N}\right\}$ and $N$ simulated values with the $i^{\text {th }}$ data point $x_{i}$ correspond to the simulated values $y\left(x_{i} ; \vec{a}\right)$. We wanted to compare the measured $\left\{y_{1}, \ldots, y_{N}\right\}$ with simulated $\left\{y\left(x_{i} ; \vec{a}\right), i=1, \ldots, N\right\}$ and find the optimal estimator of the parameter vector $\vec{a}=\left\{a_{1}, \ldots, a_{K}\right\}$. Using a first-order Taylor expansion, the linear approximation of the nonlinear regression can be expressed as

$$
y_{i}=y\left(x_{i} ; \vec{a}(0)\right)+\sum_{k=1}^{K}\left[\frac{\partial y\left(x_{i} ; \vec{a}\right)}{\partial a_{k}}\right]_{\vec{a}=\vec{a}(0)}\left(a_{k}-a_{k}(0)\right)+\varepsilon_{i}(0),
$$

where $\varepsilon_{i}(0)$ is an approximation error that we want to minimize. ${ }^{8}$ By re-parameterization, the model can be expressed as

$$
y_{i}(0)=\sum_{k=1}^{K} D_{i k}(0) \beta_{k}(0)+\varepsilon_{i}(0)
$$

where

$$
D_{i k}(0)=\left[\frac{\partial y\left(x_{i} ; \vec{a}\right)}{\partial a_{k}}\right]_{\vec{a}=\vec{a}(0)},
$$
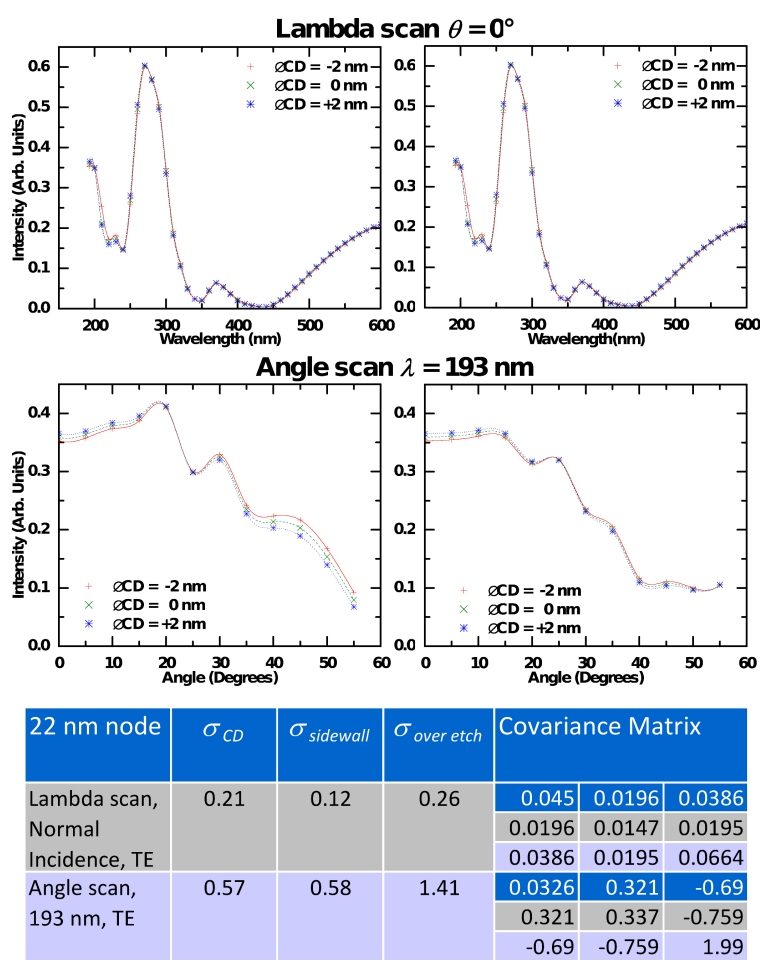

Figure 2. Transverse-electric (TE) and transverse-magnetic scans for different sidewall angles varied by $0.5^{\circ}$. The separation of the curves in the lower graphs demonstrates that the angle scans are more sensitive to $2 \mathrm{~nm}$ changes in $\mathrm{CD}$ than lambda (wavelength) scans. Relatively high cross-correlations among the parameters in angle scans (shown mathematically in the covariance matrix at bottom) result in smaller uncertainties through lambda scanning instead, despite the apparently poor sensitivity from the graphs at top. $\lambda$ : Wavelength. $\sigma_{C D}, \sigma_{\text {sidewall, }}$

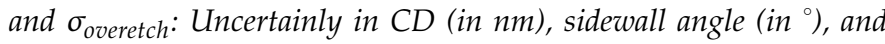
depth of the hole, which-if not perfect-over etches into another layer below (in $\mathrm{nm}$ ).

with $\beta_{k}(0)=a_{k}-a_{k}(0)$ and $y_{i}(0)=y_{i}-y\left(x_{i} ; \vec{a}(0)\right) .{ }^{8} \operatorname{Re}-$ ferring to the regression model in equation (1), we can treat prior information on $\beta_{1}$ as another 'data point. ${ }^{\prime 9}, 10$ We then get a new $\hat{\beta}^{\#}(0)=\left(D^{*}(0)^{T} V^{*}-1(0)\right)^{-1} D^{*}(0)^{T} V^{*}-1 Y^{*}(0)$ and parameter estimates $\hat{\vec{a}}_{k}^{\#}=\hat{\beta}^{\#}(0)+a_{k}(0)$ for $k=1, \ldots, K$, which now include the a priori information. (The variancecovariance matrix of the experimental values is denoted by $V=\operatorname{diag}\left[\sigma_{1}^{2}, \sigma_{2}^{2}, \cdots, \sigma_{N}^{2}\right]$.) We then obtain new uncertainties on the parameter estimates from the variance matrix, as before.

Using this knowledge, we applied the Bayesian embeddedmetrology approach to the silicon target used in Figure 1. Table 1 shows the best-fit values and uncertainties for the regression 
Table 1. Chart showing optical CD (OCD) measurements with and without embedded atomic-force microscopy (AFM) reference metrology. The

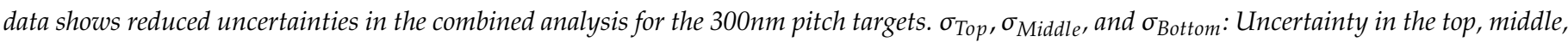
and bottom.

\begin{tabular}{|l|c|c|c|}
\hline & $\begin{array}{c}\text { OCD } \\
\text { Fitting }\end{array}$ & AFM & $\begin{array}{c}\text { OCD with } \\
\text { AFM }\end{array}$ \\
\hline Top & 120 & 119.2 & 121 \\
\hline Middle & 112 & 117.3 & 115 \\
\hline Bottom & 143 & 132.8 & 141 \\
\hline
\end{tabular}

\begin{tabular}{|l|c|c|c|}
\hline & $\begin{array}{c}\text { OCD } \\
\text { Fitting }\end{array}$ & AFM & $\begin{array}{c}\text { OCD with } \\
\text { AFM }\end{array}$ \\
\hline$\sigma_{\text {Top }}$ & 1.05 & 0.75 & 0.35 \\
\hline$\sigma_{\text {Middle }}$ & 1.58 & 0.75 & 0.60 \\
\hline$\sigma_{\text {Bottom }}$ & 0.78 & 0.75 & 0.42 \\
\hline
\end{tabular}

analysis, with and without embedded atomic-force-microscopy (AFM) reference metrology. ${ }^{11,12}$ The data shows a change in the mean values as well as an improvement in the uncertainties. The embedded reference measurement data pulls the optical CD scatterfield results toward the AFM-measured values. The resulting uncertainties from the combined measurements are lower than for each of the individual results.

The statistical approach described here allows researchers to bring together different measurement techniques that may each have their own best attributes, such as excellent bottomwidth response versus sensitivity to sidewall changes or high-throughput versus accuracy. It allows us to combine the uncertainties of two or more single measurements into an uncertainty that is an improvement over individual values. In the future, we will focus on combining results from multiple referencemetrology tools, which may may also yield improvements.

The authors would like to thank Pete Lipscomb and Michael Bishop of International SEMATECH for wafer fabrication. We are grateful to Ron Dixson for AFM reference metrology and to Thomas Germer and Alan Heckert of the National Institute of Standards and Technology (NIST) for RCWA model development and simulation support.

\section{Author Information}

\section{Richard Silver}

NIST

Gaithersburg, MD

Richard Silver is the project leader for overlay and highresolution optical metrology. He has presented a number of papers in this and related fields. He was recently chairman for the SPIE Microlithography Metrology Inspection and Process Control Conference and is also the co-chair of the Semiconductor Equipment and Materials International (SEMI) microlithography committee.

\section{Bryan Barnes and Nien Fan Zhang \\ NIST}

Gaithersburg, MD

\section{Hui Zhou}

KT Consulting

Gaithersburg, MD

\section{References}

1. R. M. Silver, B. Barnes, R. Attota, J. Jun, M. Stocker, E. Marx, and H. Patrick, Scatterfield microscopy to extend the limits of image-based optical metrology, Appl. Opt. 46 (20), pp. 4248-4257, 2007.

2. P. Boher, M. Luet, T. Leroux, J. Petit, P. Barritault, J. Hazart, and P. Chaton, Innovative photo-goniometry method for CD metrology, Proc. SPIE 5375, pp. 1302-1314, 2004. doi: $10.1117 / 12.533426$

3. R. M. Silver, T. Germer, R. Attota, B. Barnes, B. Bunday, J. Allgair, E. Marx, and J. Jun, Fundamental limits optical critical dimension metrology: a simulation study, Proc. SPIE 6518, p. 65180U, 2007. doi:10.1117/12.716604

4. R. M. Silver, B. Barnes, A. Heckert, R. Attota, R. Dixson, and J. Jun, Angle resolved optical metrology, Proc. SPIE 6922, p. 69221M, 2008. doi:10.1117/12.777131

5. B. M. Barnes, L. P. Howard, J. Jun, P. Lipscomb, and R. M. Silver, Zero-order imaging of device-sized overlay targets using scatterfield microscopy, Proc. SPIE 6518, p. $65180 \mathrm{~F}, 2007$. doi: $10.1117 / 12.716457$

6. T. A. Germer, Modeling the effect of line and trench profile variation on scatterometry measurements, Proc. SPIE 6815, p. 65180Z, 2007. doi:10.1117/12.704246

7. R. M. Silver, T. Germer, R. Attota, E. Marx, M. Davidson, B. Barnes, J. Jun, and R. Larrabee, Fundamental optical critical dimension (OCD) limits: A simulation-based study, Tech. Transfer 06044749A-TR, SEMATECH, 2006.

8. J. Neter, W. Wasserman, and M. Kutner, Applied Linear Regression Models, Richard D. Irwin, Inc., Homewood, IL, 1983.

9. A. Gelman, J. B. Carlin, H. S. Stern, and D. B. Rubin, Bayesian Data Analysis, Chapman \& Hall/CRC, Boca Raton, FL, 2000.

10. G. H. Golub and C. F. Van Loan, Matrix of Computations, $2^{\text {nd }}$ ed., The Johns Hopkins Press, Baltimore, MD, 1989.

11. R. Dixson, J. Fu, N. Orji, R. Allen, and M. Cresswell, CD-AFM reference metrology at NIST and SEMATECH, Proc. SPIE 5752, pp. 324-336, 2005. doi:10.1117/12.601972 12. R. M. Silver, N. F. Zhang, B. Barnes, H. Zhou, A. Heckert, R. Dixson, T. A. Germer, and B. Bunday, Improving optical measurement accuracy using multi-technique nested uncertainties, Proc. SPIE 7272, p. 727202, 2009. doi: $10.1117 / 12.816569$ 